\title{
A NEW CHAOTIC ATTRACTOR FROM 2D \\ DISCRETE MAPPING VIA BORDER-COLLISION \\ PERIOD-DOUBLING SCENARIO
}

ZERAOULIA ELHADJ

Received 12 April 2005

The following map is studied: $(x, y) \rightarrow\left(1+a\left(|x|-y^{2}\right)+y, b x\right)$. It is proved numerically that this model can display two different chaotic attractors, one is new and the other is a Lozi-type attractor. The new chaotic attractor is allowed via a border-collision perioddoubling scenario, which is different from the classical period-doubling bifurcation.

\section{Introduction}

The discreet mathematical models are gotten directly via scientific experiences, or by the use of the Poincaré section for the study of a continuous model. One of these models is the Henon map. Many papers have described chaotic systems, one of the most famous being a two-dimensional discrete map which models the original Henon map [3, 4, 5, 7, 8]. Moreover, it is possible to change the form of the Henon map for obtaining others chaotic attractors $[2,6]$, this type of applications is used in secure communications using the notions of chaos.

The Lozi map is 2D noninvertible iterated map proposed by Lozi [6] as follow:

$$
(x, y) \longrightarrow(1-a|x|+y, b x)
$$

This model gives a chaotic attractor called Lozi attractor and its shape is resembled to the one shown in Figure 2.1.

\section{The proposed model}

In this article, we essentially study the following modified Lozi map:

$$
(x, y) \longrightarrow\left(1+a\left(|x|-y^{2}\right)+y, b x\right) .
$$

We show numerically that the new model (2.1) can display two different chaotic attractors; including the classical Lozi-type attractor as shown in Figure 2.1 to Figure 4.2. 


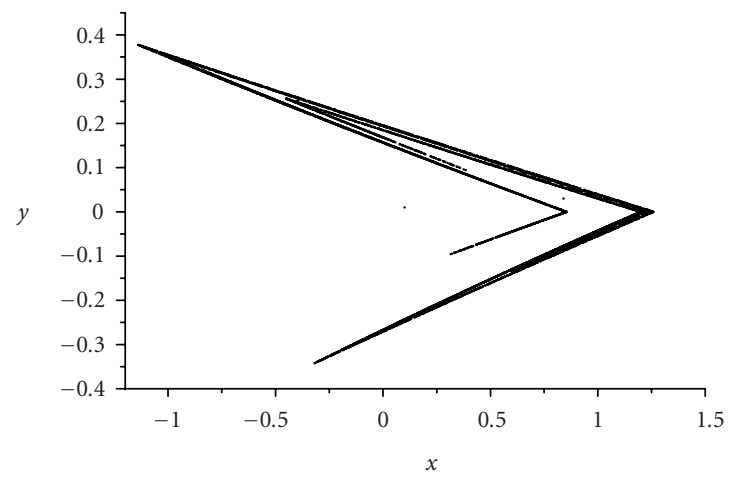

Figure 2.1. A Lozi-type chaotic attractor obtained from system (2.1) for $a=-1.8, b=0.3$.

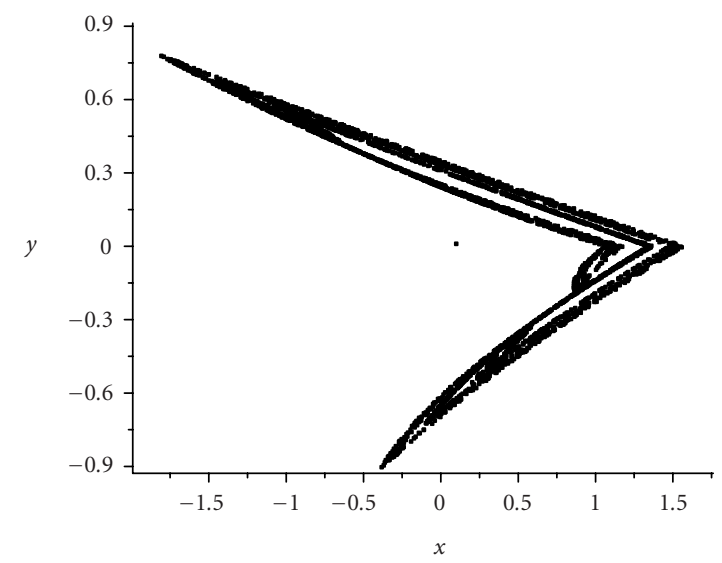

Figure 2.2. A Lozi-type chaotic attractor obtained from system (2.1) for $a=-1.8, b=0.5$.

\section{Comparison with the Lozi system}

The system (2.1) has the same complexity as the Lozi system (1.1), they are both twodimensional discrete noninvertible maps. However, the two models are topologically not equivalent because the Lozi system (1.1) is a piecewise-linear map, but model (2.1) is a nonlinear system. In addition; it can be rigorously proved that a non-singular homeomorphism that transforms each system to other does not exist. The proof needed some straightforward but tedious algebra which leads to a system of algebraic equations without solutions.

\section{Route to chaos}

It is well known that while varying the parameter $a$, the Hénon attractor is obtained via a period-doubling bifurcation route to chaos as a typical future [3], unless for Lozi map, no 


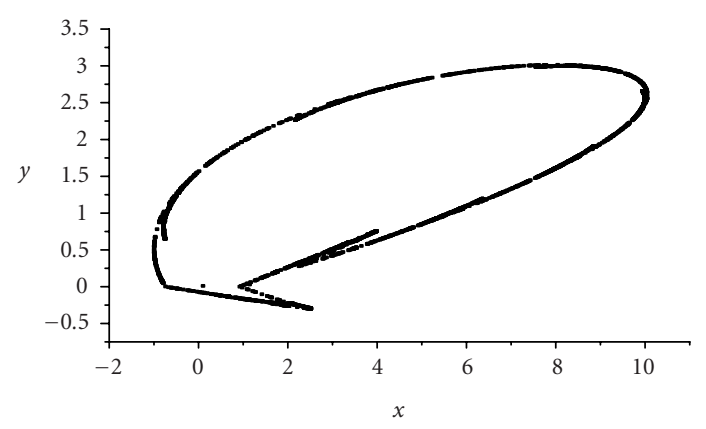

Figure 4.1. A typical orbit of system (2.1) obtained for $a=1.35, b=0.3$.

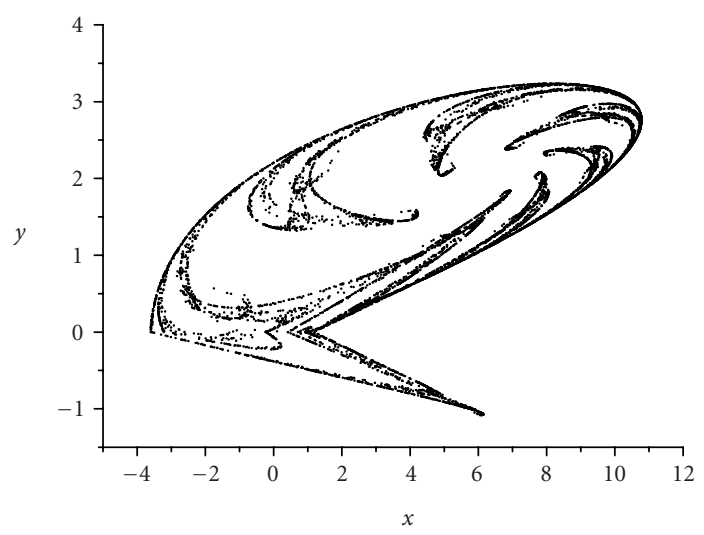

Figure 4.2. The new chaotic attractor obtained for $a=1.4, b=0.3$.

period doubling route to chaos is allowed, and the attractor goes directly from a bordercollision bifurcation developed from a stable periodic orbit [4], and for the same case the new chaotic attractor given by system (2.1) is obtained from a border-collision perioddoubling bifurcation scenario [1]; this scenario (see Figure 4.3) is formed by a sequence of pairs of bifurcations, whereby each pair consists of a border-collision bifurcation and a pitchfork bifurcation. Thus, the three chaotic attractors go via different and distinguishable route to chaos as a typical future.

\section{Conclusion}

This paper reports the finding of a new two-dimensional discrete chaotic attractor obtained via direct modification of the Lozi mapping. The new chaotic attractor is allowed via a border-collision period-doubling scenario. More detailed analysis about the structure, dynamics and bifurcations of the new system (2.1) will be provided in near future. 


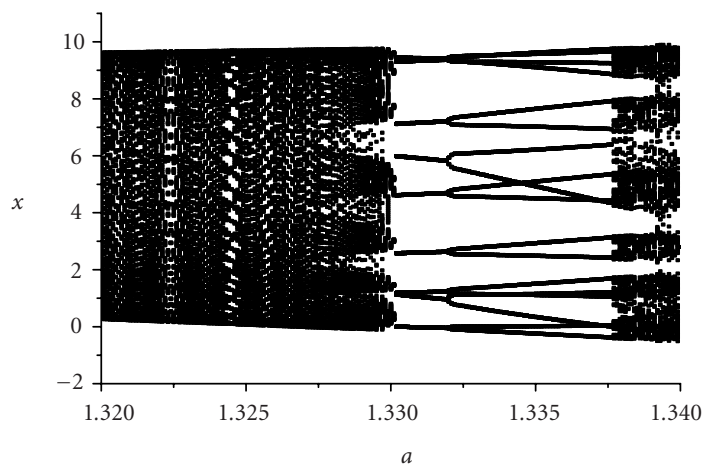

Figure 4.3. Border-collision period-doubling scenario route to chaos observed for system (2.1).

\section{References}

[1] V. Avrutin and M. Schanz, Border-collision period-doubling scenario, Phys. Rev. E (3) 70 (2004), no. $2,026222,11$.

[2] M. A. Aziz-Alaoui, C. Robert, and C. Grebogi, Dynamics of a Hénon-Lozi-type map, Chaos Solitons Fractals 12 (2001), no. 12, 2323-2341.

[3] M. Benedicks and L. Carleson, The dynamics of the Hénon map, Ann. of Math. (2) 133 (1991), no. $1,73-169$.

[4] Y. Cao and Z. Liu, Strange attractors in the orientation-preserving Lozi map, Chaos Solitons Fractals 9 (1998), no. 11, 1857-1863.

[5] M. Hénon, A two-dimensional mapping with a strange attractor, Comm. Math. Phys. 50 (1976), no. $1,69-77$.

[6] R. Lozi, Un attracteur étrange du type attracteur de Hénon, J. Phys. Colloque C5 39 (1978), Supplément au no. 8, 9-10 (French).

[7] F. R. Marotto, Chaotic behavior in the Hénon mapping, Comm. Math. Phys. 68 (1979), no. 2, 187-194.

[8] M. Misiurewicz, Strange attractors for the Lozi mappings, Nonlinear Dynamics (Internat. Conf., New York, 1979) (R. G. Heman, ed.), Ann. New York Acad. Sci., vol. 357, New York Academy of Sciences, New York, 1980, pp. 348-358.

Zeraoulia Elhadj: Department of Mathematics, University of Tébéssa, 12000 Tébéssa, Algeria E-mail address: zelhadj12@yahoo.fr 


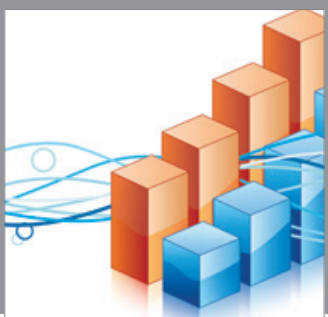

Advances in

Operations Research

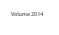

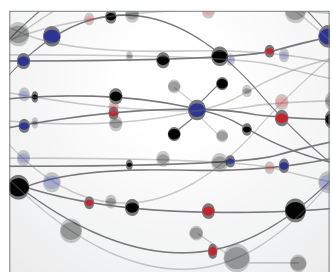

\section{The Scientific} World Journal
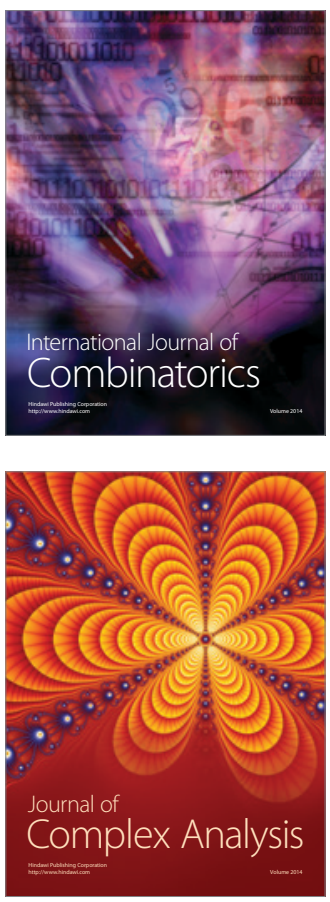

International Journal of

Mathematics and

Mathematical

Sciences
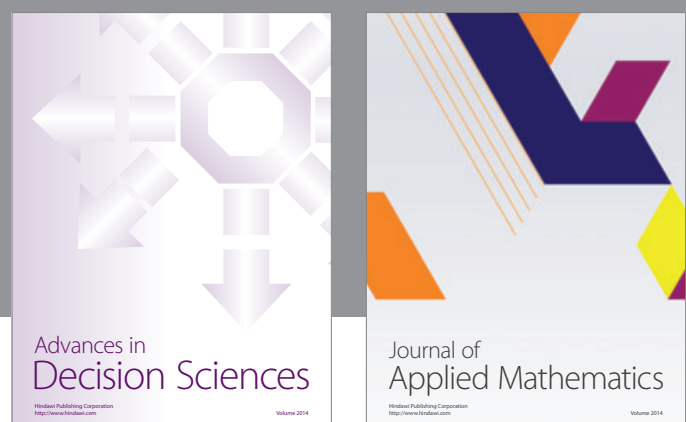

Journal of

Applied Mathematics
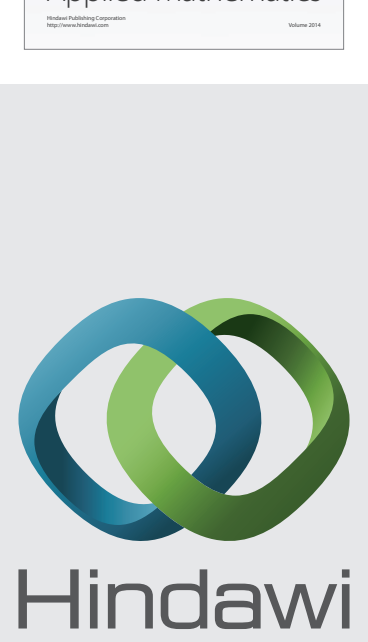

Submit your manuscripts at http://www.hindawi.com
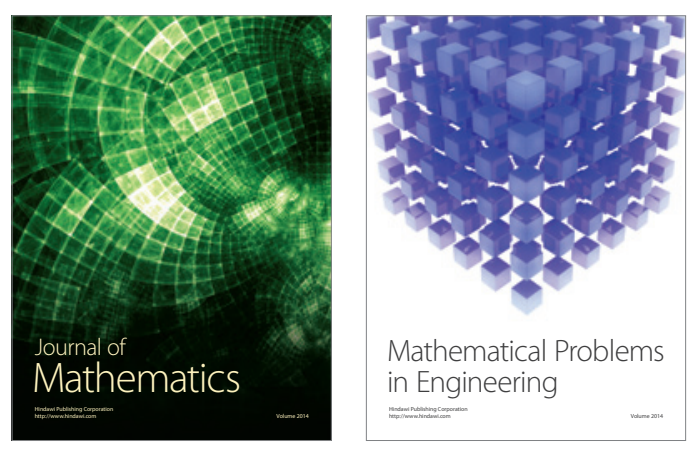

Mathematical Problems in Engineering
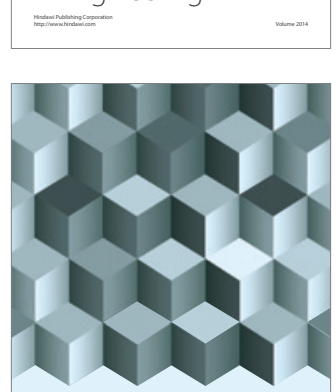

Journal of

Function Spaces
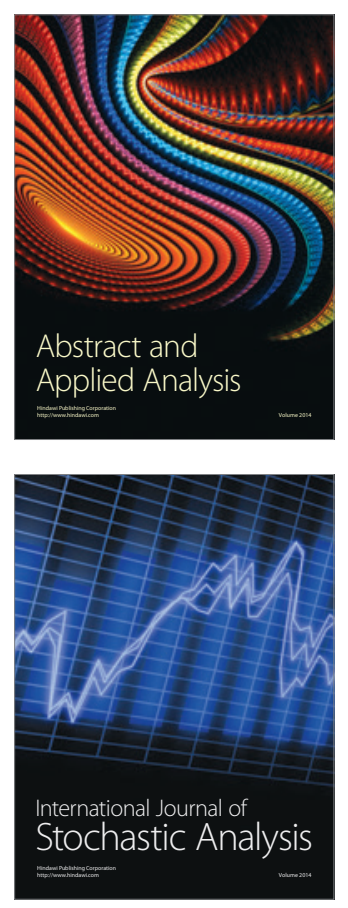

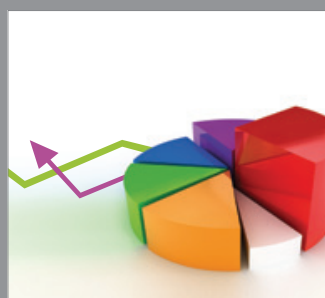

ournal of

Probability and Statistics

Promensencen
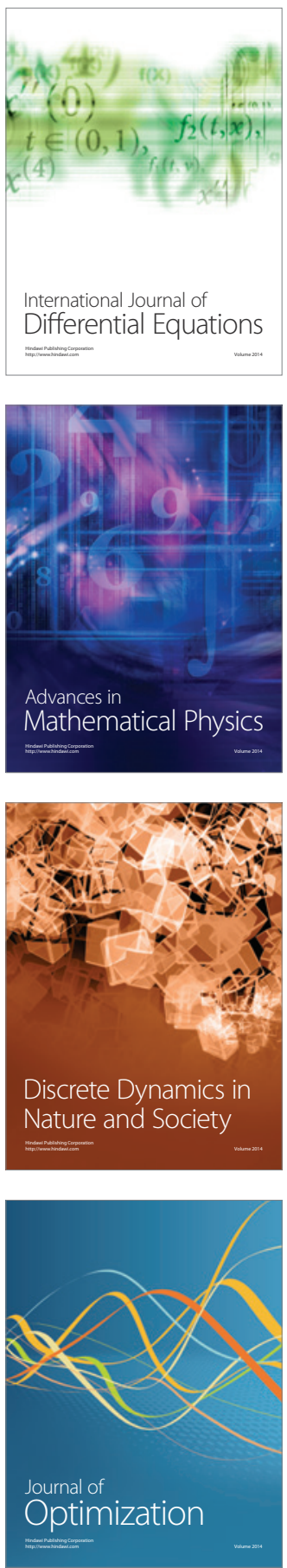